\title{
UAV BASED BRDF-MEASUREMENTS OF AGRICULTURAL SURFACES WITH PFIFFIKUS
}

\author{
G. J. Grenzdörffer*, F. Niemeyer ${ }^{\text {a }}$
}

${ }^{\text {a }}$ Chair for Geodesy and Geoinformatics, Rostock University,18059 Rostock, Germany - [goerres.grenzdoerffer]

[frank.niemeyer]@uni-rostock.de

Commission I, WG I/V

KEY WORDS: UAVs, Photogrammetry, BRDF, camera calibration, four vision camera

\begin{abstract}
:
BRDF is a common problem in remote sensing and also in oblique photogrammetry. Common approaches of BRDF-measurement with a field goniometer are costly and rather cumbersome. UAV's may offer an interesting alternative by using a special flight pattern of oblique and converging images. The main part of this paper is the description of a photogrammetric workflow in order to determine the anisotropic reflection properties of a given surface. Due to the relatively low flying heights standard procedures from close range photogrammetry were adopted for outdoor usage. The photogrammetric processing delivered automatic and highly accurate orientation information with the aid of coded targets. The interior orientation of the consumer grade camera is more or less stable. The radiometrically corrected oblique images are converted into ortho photos. The azimuth and elevation angle of every point may then be computed. The calculated anisotropy of a winter wheat plot is shown. A system four diagonally-looking cameras (Four Vision) and an additional nadir looking camera is under development. The multi camera system especially designed for a MicroUAV with a payload of min $1 \mathrm{~kg}$. The system is composed of five industrial digital frame cameras (1.3 Mpix CCD-chips, $15 \mathrm{fp} / \mathrm{s})$ with fixed lenses. Also special problems with the construction of a light weight housing of the multi camera solution are covered in the paper.
\end{abstract}

\section{INTRODUCTION}

\subsection{Why do we need BRDF-measurements ?}

Multi-angular remote sensing has became a hot topic after the non-Lambert characteristic of the Earth's surface was accepted popularly, e.g. von Schönermark et al., 2004. For agricultural and forestry applications, a reliable derivation of biophysical variables from remote sensing data such as chlorophyll content, biomass, etc. is very important. This data can for example serve as a key input in the calculation of site specific fertilizer measures or to calculate the vitality of forest stands. However, for the automated calculation of these parameters, a calibrated sensor, the consideration of atmospheric effects, including the bidirectional reflectance properties of plant surfaces (BRDF) is required. The latter in particular is complicated to determine, expensive and requires a large computational effort, thus preventing a quantitative near-real time use of inexpensive remote sensing data.

In recent years the determination of the BRDF-function of vegetation surfaces in the field was done either with field spectrometers mounted on a field goniometer or on top of a mobile goniometer system. These instruments are generally very costly and in most instances difficult to handle in a mobile environment. Furthermore conventional field spectrometer measurements are commonly taken under "perfect conditions", which generally means clear blue sky, Sandmeier \& Itten, 1999.

The spectral properties of agricultural crops change significantly with the observed distance to the object or more precisely with the instantaneous field of view or the foot print of a sensor element. This phenomenon is related to the fact, that the spectral properties of a single leaf are significantly different than the crop canopy where not only the leafs are visible, but also the soil background and the shade in between different leafs, Jones and Vaughan, 2010. This up scaling problem of common field goniometers from leaf level to canopy level is difficult to tackle and no satisfying solutions were found so far, Schneider et al., 2006.

Therefore a UAV-based approach shall be an interesting alternative for BRDF-measurements. However the UAVapproach presented is not to bring a light weight field spectrometer in the air to extend the field goniometer approach to a larger scale. Also our approach is not eliminate the BRDFeffects statistically, e.g. presented by Lelong et al., 2008. Instead our approach is more influenced by the idea of photogrammetric self calibration. The final goal of our research is a combination of oblique looking (multispectral) cameras and software that is able to analyse the anisotropic spectral behaviour of any object. The anisotropic properties shall then used for either radiometric image correction or otherwise serve as an additional source of information for the determination of special object properties, such as the crop vitality. However some special problems have to be solved. One of the most demanding problems is the precise and, if possible, automated georeferencing of the UAV-imagery.

\subsection{UAV and oblique imagery}

Due to regulations micro-UAV's are commonly bound to applications within the line of sight. The maximum elevation allowed differs from country to country but it normally does not exceed $300 \mathrm{~m}$. At this elevation a wide angle camera image covers an area of approximately $200 \times 300 \mathrm{~m}$. An extension of the ground coverage per image is possible by using an oblique camera. An oblique view covers a larger area, though not at the same scale throughout the image, Grenzdörffer et al, 2008.

Oblique-looking images of vegetation surfaces provide an additional advantages of are a lower proportion of the soil 
background and a lesser amount of shadows in relation to the vegetation signal. This improves the signal-to-noise ratio and a significant plant signal can be seen phenologically much earlier than in a comparable nadir shot. However, images acquired from a single oblique direction are much more effected by the BRDF. This means that the reflection of the vegetation surface strongly depends on the viewing direction and the geometric relationship between the sun and the observer (camera). Only with a synchronous recording of four cameras with $90^{\circ}$ viewing directions, interfering effects (BRDF) can be computed, which otherwise makes the automated derivation of vegetation parameters almost impossible.

\section{DETERMINATION OF BRDF - PHOTOGRAMMETRIC WORKFLOW}

The developed workflow presented in this paper consists of eight steps, which be discussed in the following paragraphs

1. Hemispheric flight planning

2. Layout of coded targets

3. Aerial survey with converging images

4. Automatic (aero)triangulation incl. measurement of the interior orientation

5. Radiometric calibration and vignetting reduction

6. Ortho rectification of imagery

7. Determination of anisotropy factor

Future work will extend this workflow with additional processing steps.

8. Comparison with field spectrometer values

9. Modeling and image correction

\subsection{Hemispheric flight pattern for BRDF-Measurements}

In order to determine the anisotropy of a given surface a complex flight pattern for the UAV was developed. The hemispheric flight pattern is based on the idea, that the BRDF shall be determined from several directions, similarly to a field goniometer. The flight pattern acquires images at $30^{\circ}$-steps in the azimuth direction and also every $15^{\circ}$ in the elevation, respectively the zenith angle. The developed flight pattern (Figure 1) takes 60 images. The flight pattern was developed for the trial plots of the agricultural extension site of Rostock University. The covered trial fields are approx. $10 \times 10 \mathrm{~m}$. The radius of the flight pattern is $25 \mathrm{~m}$ in extend and elevation. It takes the MD 4-1000 approx. 15 minutes to fly the whole flight pattern.

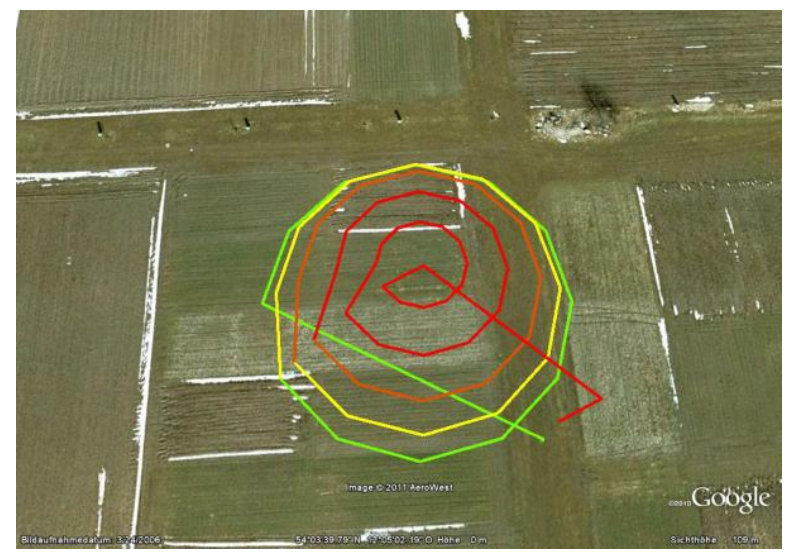

Figure 1: Flight pattern for BRDF-measurements
For the BRDF measurements images from the principal plane as well as from the cross plane are most important. During the course of the day and year the sun always changes its position relative to the observer. In order to maintain the predefined flight pattern for every occasion image acquisition has to take place at different day times. Figure 2 demonstrates this for two different BRDF-surveys.

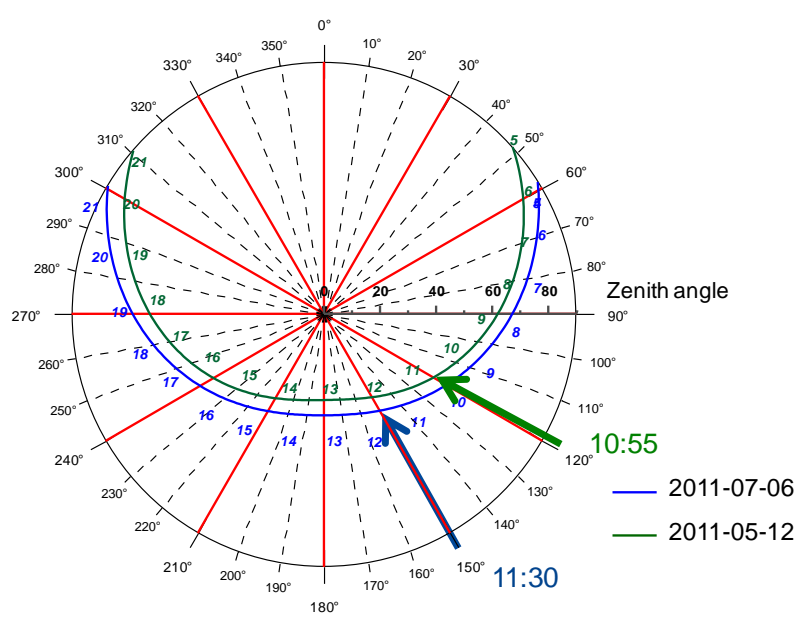

Figure 2: Illumination properties and selected flight times during the test flights

\subsection{Layout of coded targets}

Ground control points for airborne photogrammetric geo correction are commonly single circular or quadratic points. Unfortunately they do not allow for an automatic recognition. This is only possible with so called coded targets. Coded targets are composed of a defined pattern of points or other automatically recognizable features. They provide the possibility of reliable detection and localisation in a textured image and offer precise sub pixel centre coordinates calculation. For a precise pattern recognition a coded target must have a certain minimum size of 20 - 100 pixel. For airborne applications with a GSD of $10 \mathrm{~cm}$ or more coded are generally not used, because the targets on the ground would become too large. For UAV flights at low altitudes with a GSD of $1-2 \mathrm{~cm}$ however coded targets are an interesting alternative for rapid and automatic image geocoding.

For close range photogrammetry applications in the lab retro reflecting coded targets are commonly used, because they provide a perfect determination of significant marked targets and other (white) spots which are not interest. However retro reflecting targets require a flash illumination which is not feasible for an outdoor application. For outdoor applications the question arises to use either black targets on white ground or black targets on a white background. The latter option is much easier to realize, because they can be plotted on every plotter without using too much ink. Unfortunately they become smaller and smaller on an image under bright imaging conditions, as illustrated on Figure 3. This observation is most probably due to the fact that the cameras used are not of best quality. 


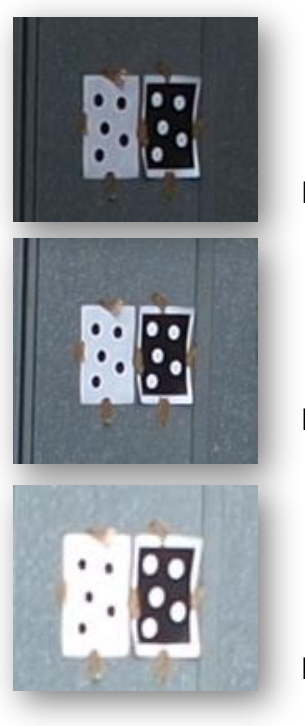

Dark conditions

Normal conditions

Bright conditions

Figure 3: Visibility of coded targets under different illumination conditions

Nevertheless the over exposure of white blobs on the dark background allows for smaller targets, than the mathematical GSD would assume. The GSD of our flights with maximum flying height of $25 \mathrm{~m}$ and a slant distance to the coded targets of no more than $35 \mathrm{~m}$, does not exceed $0.9 \mathrm{~cm}$. Sub pixel accuracy through automatic point measurements of a circular blob, e.g. by using a ellipse operator requires a minimum diameter of the target of 5 pixel, Luhmann et al, 2006. Therefore circular white codes of $4 \mathrm{~cm}$ in diameter were put on a black back cover. For outdoor use the coded targets had to be laminated and stabilized through a hard plastic back cover. The total size of a coded target is approximately $60 \times 40 \mathrm{~cm}$.

In the field the mobile targets were laid out around the test site, Figure 4. Furthermore single targets were put in the corners and their position was measured with a Leica 1230 RTK-GPS receiver.

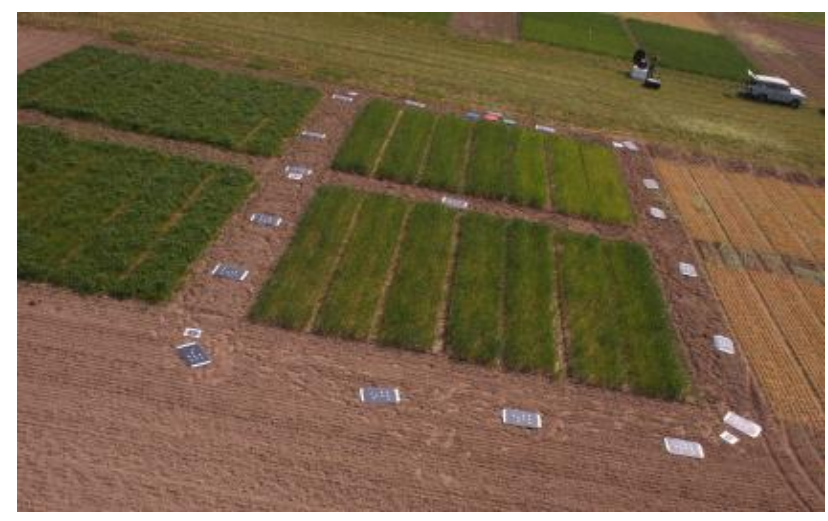

Figure 4: Test site with calibration targets (2011-05-12)

\subsection{Aerial survey with converging images}

The test flight were carried out with a Microdrones MD4-1000 UAV. The UAV has a 2-axis active stabilised mount which ca point in any desired direction. The UAV is equipped with an Olympus E-P2 camera. The 12.3 megapixel camera has a CMOS-sensor and a fixed lens of nominal $17 \mathrm{~mm}$.
The aerial surveys were carried out at the field trial station of Rostock University. Winter wheat with different fertilizer levels is grown on the examined test plots. A total of four test flights were carried out:

- 23.04.2011 (clear sky, nice BRDF, but white targets with black dots didn't work)

- 11.05. and 12.05.2011 (overcast sky, little BRDF visible)

- 06.07.2011 (clear sky, partial flight for TV-media images)

The following presented empirical data is based to the test flights of 11.5.2011 and 12.05.2011, if not noted otherwise.

For practical reasons it is important to see how good the predefined flight pattern was executed by the UAV. Figure 5 gives a practical impression for the test flight on 12.05.2011.

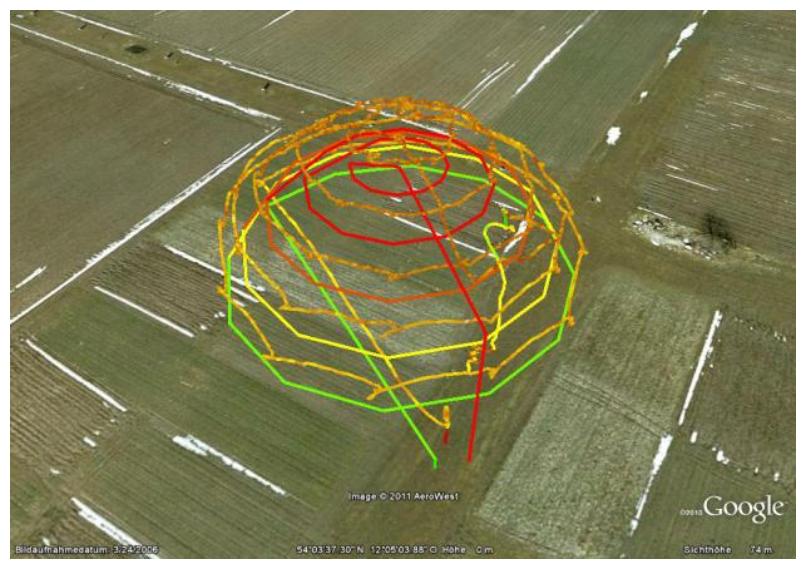

Figure 5: Comparison of planned and realized flight pattern

Generally speaking the UAV executed the flight pattern quite nicely, taking the low wind conditions during the flight into account. However two relevant issues were observed:

1. The UAV must be hovered precisely to the starting elevation of the flight pattern, otherwise the UAV will execute the survey with a height offset.

2. The UAV was preset to stop at every waypoint. This caused additional stabilisation manoeuvres of the UAS and the stabilized mount, thus creating more instability and possible sources of error. Future flights will therefore be done in a continuous mode.

\subsection{Automatic (aero)triangulation incl. measurement of the interior orientation}

From the 60 input images only 33 images could be triangulated with the photogrammetric software Australis 7 automatically, Figure 6. Accuracy of the aero triangulation of the remaining images has an internal sigma0 of $0.49 \mu \mathrm{m}(=0.11$ Pixel). After the aero triangulation was carried out in an arbitrary internal coordinate system a transformation into the geodetic coordinate system (UTM 33) was carried out through a 3D Helmert transformation with the precisely measured ground control points. 


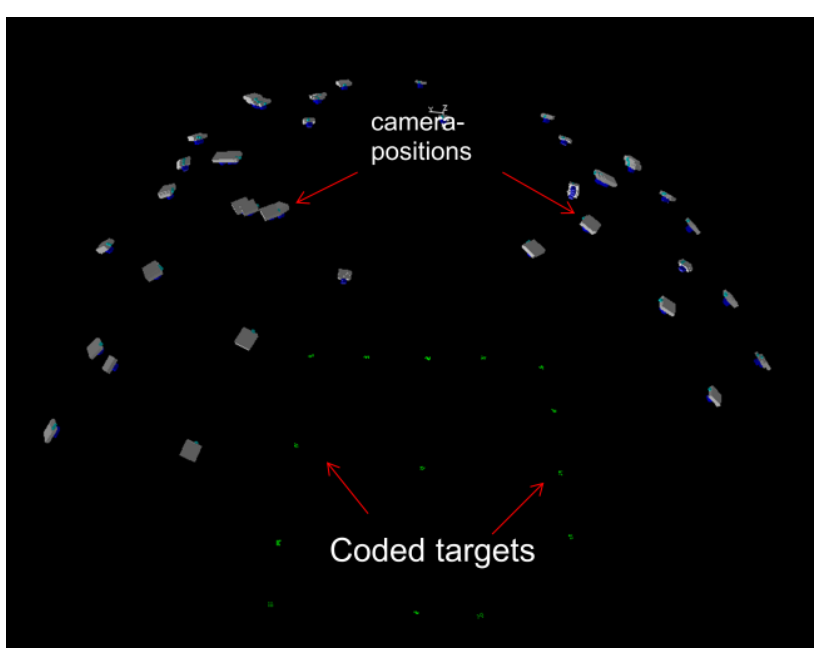

Figure 6: BRDF-flight 2011-05-12 - Reconstructed camera positions

Targets around the hot spot and the sun glint position could not be identified automatically due to the necessary plastic lamination cover of the targets. Therefore no exterior orientation for images acquired at the hot spot position could be determined.

Since the interior orientation is computed along with the exterior orientation, the parameters could be compared with a previous lab calibration, Table 1 and Figure 7.

\begin{tabular}{|lll|}
\hline & $\begin{array}{l}2011-05-12 \\
\text { (Field calibration) }\end{array}$ & $\begin{array}{l}2011-04-01 \\
\text { (Lab calibration) }\end{array}$ \\
\hline$c$ & $16.701 \mathrm{~mm}$ & $16.625 \mathrm{~mm}$ \\
$x p$ & $0.027 \mathrm{~mm}$ & $0.040 \mathrm{~mm}$ \\
$y p$ & $0.029 \mathrm{~mm}$ & $-0.113 \mathrm{~mm}$ \\
\hline
\end{tabular}

Table 1: Parameters of the interior orientation of Olympus E-P2 determined in field and laboratory

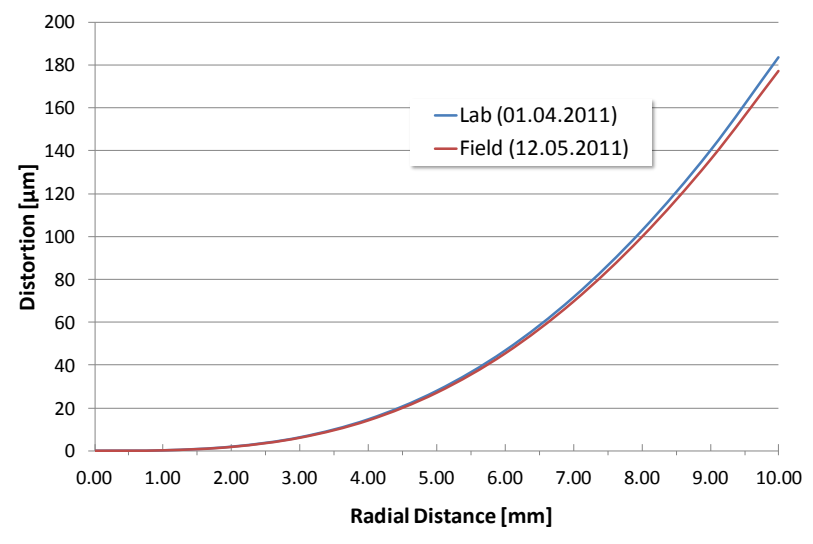

Figure 7: Olympus PE2 - comparison of radial distortion measurements (in field and laboratory)

The comparison reveals that the interior orientation is not perfectly stable. While the radial distortion is quite similar, the focal length and the principal point has undergone significant changes. Nevertheless these differences are quite common for consumer grade digital cameras.

\subsection{Radiometric calibration and vignetting reduction}

The geometric and radiometric properties of the camera were examined quite intensively, table 2 .

\begin{tabular}{ll}
\hline Geometric Parameters & Radiometric Parameters \\
\hline interior orientation & colour artefacts \\
stability of the lens & linearity, dynamic range \\
resolving power (MTF) & noise (SNR) \\
& Vignetting \\
\hline
\end{tabular}

Table 2. Evaluated radiometric and geometric properties of Olympus EP-2

The lens reveals a sharp drop in image quality at the edges. The determination of the vignetting of the lens in relation to the $f$ stop is quite important for the further radiometric processing, because it directly interferes with the BRDF. The vignetting of the camera was determined by a defocused photograph an evenly illuminated white sheet of paper. The vignetting correction was carried out with the freeware "vignetting reducer $1.7^{\prime \prime}$.

\subsection{Ortho rectification of imagery}

The ortho rectification is a necessary step in the process chain, because the parameters of the exterior orientation determine only the azimuth and zenith angle of the image centre directly. For all other image points the azimuth and zenith angle can be computed easily with the difference of the coordinates using the following formulas (1-3), see also Figure 8.

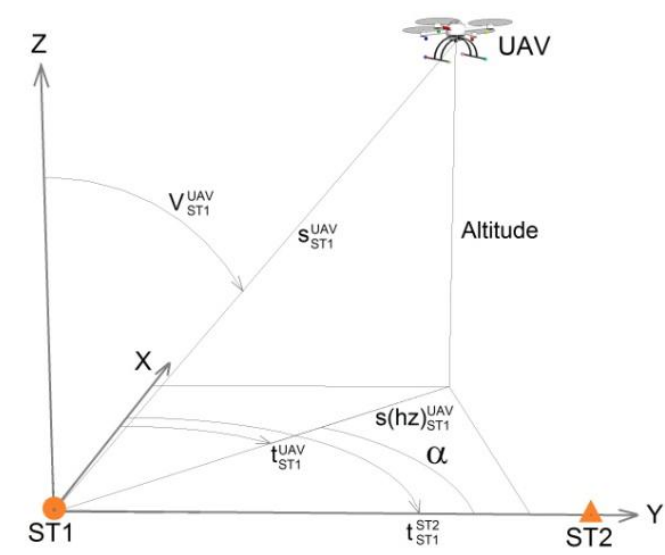

Figure 8: Azimuth and zenith angle

$$
\begin{aligned}
& A z_{S T 1}^{U A V}=\arctan \frac{\Delta Y}{\Delta X}=\frac{Y_{U A V}-Y_{S T 1}}{X_{U A V}-X_{S T 1}}+\text { Quadrant } \\
& D_{S T 1}^{U A V}=\sqrt{\Delta x^{2}+\Delta y^{2}} \\
& (V)=\arctan \left(\frac{D_{S T 1}^{U A V}}{\text { Altitude }}\right)+\text { Quadrant }
\end{aligned}
$$

The radiometrically corrected imagery was ortho rectified with Leica Photogrammetry Suite (LPS) from ERDAS. The necessary DEM of the rather flat test site is based on a rectangular grid $(10 \times 10 \mathrm{~m})$ of GPS-measurements. The accuracy of the final ortho rectified imagery in the object space is approximately $5 \mathrm{~cm}$. 


\subsection{Determination of anisotropy factor}

Due to overcast and partly cloudy conditions, the weather for anisotopy determination was quite unfavourable during the test flights on 11.05.2011 and 12.05.2011.

Winter wheat with different levels of fertilizer was grown at the selected test site of the trial field extension of Rostock University. At 11.05.2011 the winter wheat was at the Feekes growth stage 9.0 with a fully emerged flag leave. The crop height was approx. $30-40 \mathrm{~cm}$. The visible soil back ground (from a nadir looking perspective) differed between $15-40 \%$, related to the amount of $\mathrm{N}$-fertilizer applied.

In total 10 quadratic plots with $1 \times 1 \mathrm{~m}$ were selected in the ortho photos. The mean RGB-values for each plot were calculated and compared. Four of the selected plots with healthy vegetation were quite similar with a difference of $<5 \mathrm{DN}$ in the RGB values. Three other similar plots revealed rather sparse crops.

The azimuth and elevation angle was computed for every image and plot. Images taken $\pm 15^{\circ}$ off the principal plane and the cross plane were grouped together to compute the anisotropy. The results for the group of four vivid crop plots are shown in Figure 9 and Figure 10. Little anisotropy is visible in the principal plane. This is most probably due to the fact that hot spot images are missing. Despite the weather the anisotropy in the cross plane still clearly visible. The anisotropy in the forward looking direction is due to differences in the visible amount of shade and soil back ground, because it is most significant in the blue and the red band.

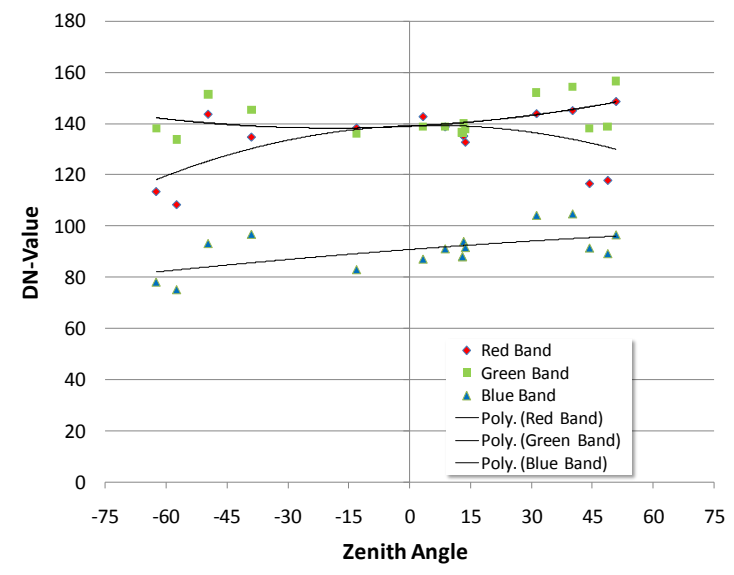

Figure 9: Anisotropic behavior of a winter wheat plot in the principal plane, image acquired 11.05.2011

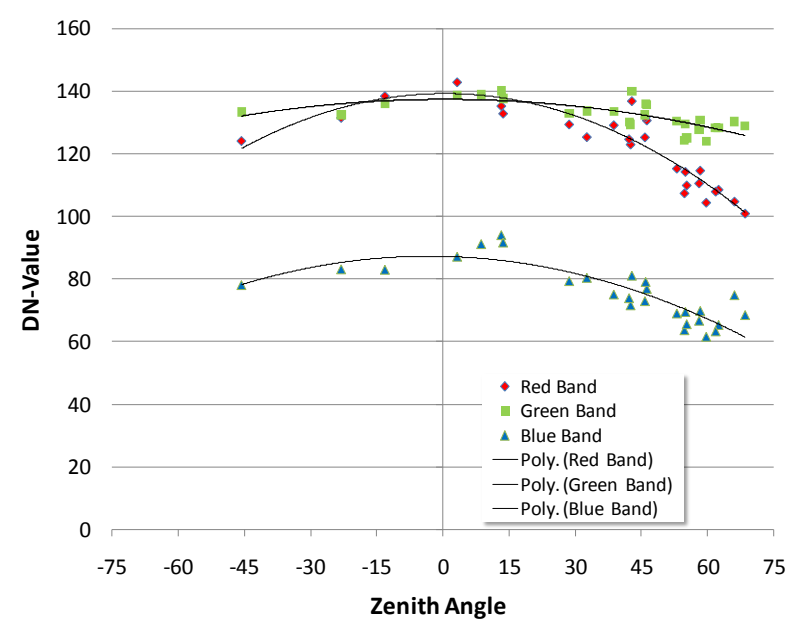

Figure 10: Anisotropic behavior of a winter wheat plot in the cross plane, image acquired 11.05.2011

Future work has to validate the preliminary results with a field spectrometer. Another focus will be on how to apply the determined anisotropy to the images and how to incorporate this additional information into the image analysis for precision farming applications.

\section{FOUR VISION CAMERA SYSTEM}

Oblique images are difficult to obtain with standard mapping cameras. To fully exploit the information from the oblique perspective, a minimum of four images from all sides have to be acquired and managed. Only multi head camera systems provide the necessary flexibility. For the professional acquisition of oblique images several companies developed multiple camera head solutions with five cameras (Petrie, 2009). This configuration is also called the "Maltese Cross" configuration. In such systems, one camera head provides a nadir view and the other four cameras provide the fixed oblique views in different directions.

While there are several medium format multi head camera systems available, e.g. Grenzdörffer, 2010, no UAV configuration has been established so far. The general constraints for the development of the "four vision" camera system for a Micro-UAV are:

- $\quad$ industrial grade programmable (video) cameras which are well suited for photogrammetric applications

- $\quad$ low weight (max. 1.000 g.) of the system

- low energy consumption

- flexible construction to allow for different viewing configurations

- on board data storage and GPS-based flight management

- $\quad$...

Figure 11 shows the most important components and the configuration of the four vision system. 


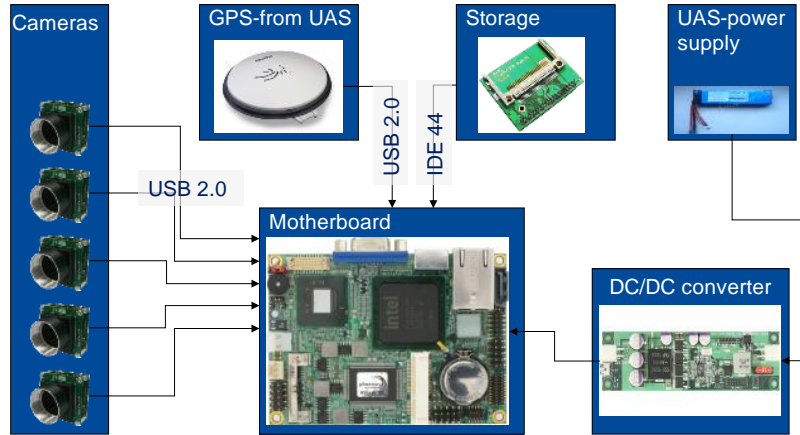

Figure 11. Components of the four vision camera system

The light weight carbon fiber construction is currently under development (Figure 12). It will weight no more than $80 \mathrm{~g}$ including a dark cover, that prevents unwanted incident sun light.

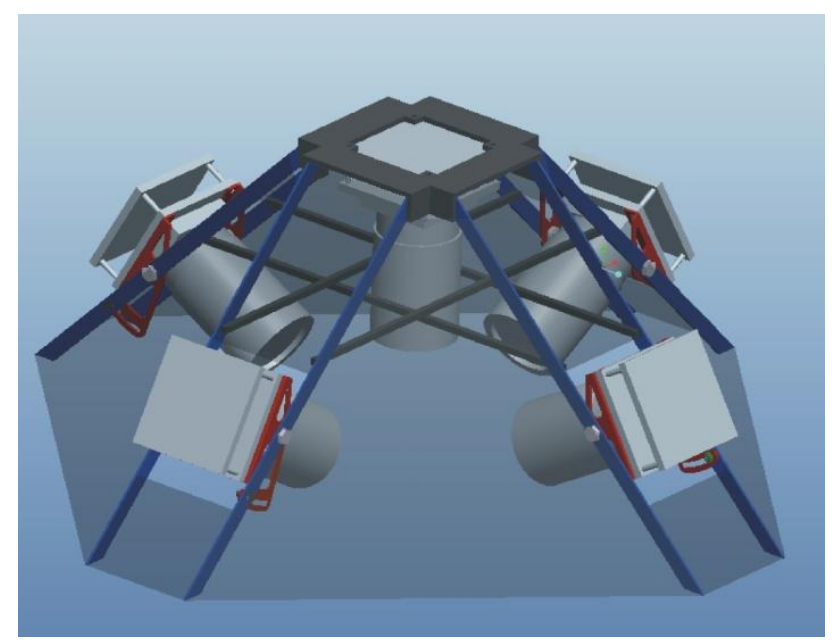

Figure 12. Construction of the camera system

\subsection{References}

Grenzdörffer G., Guretzki, M., Friedlander, I., 2008: Photogrammetric image acquisition and image analysis of oblique imagery.- Photogrammetric Record, Vol. 23, Issue 124 (Dec. 2008). 372 - 386

Grenzdörffer, G. (2010): Performance of Medium Format Digital Airborne Cameras.- EuroSDR Official Publication No 58: S. 233 - 265. ISSN 0257-0505

Jones. H.G. and Vaughan, R.A. (2010): Remote sensing of vegetation.- Oxford University Press $353 \mathrm{p}$

Luhmann, T., Robson S., Kyle, S. (2006): Close Range Photogrammetry: Principles, Techniques and Applications.- 528 p., John Wiley \& Sons

Lelong, C.C.D.; Burger, P.; Jubelin, G.; Roux, B.; Labbé, S.; Baret, F. (2008): Assessment of Unmanned Aerial Vehicles Imagery for Quantitative Monitoring of Wheat Crop in Small Plots. Sensors 2008, 8, 3557-3585. 7

Petrie, G., 2009c: Systematic Oblique Aerial Photography Using Multiple Digital Frame Cameras. Photogrammetric Engineering \& Remote Sensing, 2/2009, S. 102-107. (=http://www.asprs.org/publications/pers/2009journal/february/ highlight.pdf)

Sandmeier, S. R. and Itten I. (1999): A Field Goniometer System (FIGOS) for Acquisition of Hyperspectral BRDF Data.IEEE transactions on geoscience and remote sensing, vol. 37, no. 2, pp. 978 - 986

Schneider, T., Dorigo, W, Huber, K., Schneider, W. (2006): Field goniometer measurements for biophysical parameter retrieval in support of chris data evaluations.- Proceedings of the 4th ESA Proba/CHRIS workshop, 19th-21th of September 2006, Frascati, Italy 8 p.

von Schönermark, M.; Geiger, B.; Röser, H.P. (2004): Reflection properties of vegetation and soil, 1 st 7 ed.; Wissenschaft und Technik Verlag: Berlin, Germany, 352 p.

\subsection{Acknowledgements}

The presented research "PFIFFikus" is supported by TBI Technologie-Beratungs-Institut $\mathrm{GmbH}$ of the Ministry of Economics, Labour and Tourism Mecklenburg-Vorpommern under the grant number V230-630-08-TFMV-F041 